
\title{
$\checkmark$ Research Square \\ Determination of Significant Prognostic Factors for Maxillary Gingival Squamous Cell Carcinoma in 90 Cases
}

Yoshio Ohyama ( $\sim$ yohyama1219@gmail.com )

Shizuoka City Shizuoka Hospital https://orcid.org/0000-0002-9969-2531

Masashi Yamashiro

NTT Higashi Nihon Kanto Byoin

Yasuyuki Michi

Tokyo Medical and Dental University: Tokyo Ika Shika Daigaku

Narikazu Uzawa

Osaka University: Osaka Daigaku

Kunihiro Myo

Gunma Kenritsu Gan Center

Itaru Sonoda

Moriyama Memorial Hospital

Jun Sumino

Saitama cancer center

Chika Miura

Ebara Hospital

Miho Mizutani

NTT Higashi Nihon Kanto Byoin

Daisuke Yamamoto

Tokyo Medical and Dental University: Tokyo Ika Shika Daigaku

Kou Kayamori

Tokyo Medical and Dental University: Tokyo Ika Shika Daigaku

Tetsuya Yoda

Tokyo Medical and Dental University: Tokyo Ika Shika Daigaku

Research article

Keywords: maxillary gingival carcinoma, site of occurrence, squamous cell carcinoma, prognostic factor

Posted Date: December 3rd, 2020

DOl: https://doi.org/10.21203/rs.3.rs-116831/v1 
License: (c) (i) This work is licensed under a Creative Commons Attribution 4.0 International License. Read Full License 


\section{Abstract}

\section{Background}

Maxillary gingival squamous cell carcinoma (MGSCC) occurs rather infrequently, compared to tongue and mandibular gingival carcinomas, among the cancers of the oral cavity. Therefore, significant numbers of MGSCC cases have not been statistically analysed. The aim of this study is to clarify the prognostic factors for MGSCC.

Methods

We performed the statistical analysis of 90 MGSCC cases primarily treated in our department from 1999 to 2014.

Conclusions

The patients (male: 36 , female: 54 ) were aged between 38 and 93 years, and the mean age was 68.7 years. The number of patients in each tumour stage according to the TNM classification was as follows: T1: 15 cases, T2: 32 cases, T3: 13 cases, and T4: 30 cases. Forty-two patients were treated only by surgery, 5 only by radiotherapy, 3 by preoperative radiotherapy and surgery, and 40 patients were treated by combination therapy with preoperative chemoradiotherapy and surgery. Neck dissections were performed in 40 cases including 29 cases (11 primary and 18 secondary cases) of histopathologically diagnosed lymph node metastases. Extranodal extension was found in $74.3 \%$ cases with metastatic lymph nodes. The 5 -year overall survival rate was $81.9 \%$. In univariate analysis, the site of occurrence, stage of tumour, lymph node metastasis, and treatment contributed to the 5-year survival rate. Multivariate analysis demonstrated that the site of occurrence (posterior region) was an independent prognostic factor. Seventeen deaths occurred due to the primary disease, while three deaths were caused by other diseases.

Conclusion

The posterior region cancers, according to the classification based on site of occurrence, were independent predictors of poor 5-year overall survival rate.

\section{Background}

Maxillary gingival squamous cell carcinoma (MGSCC) occurs rather infrequently compared to mandibular gingival carcinoma. $[1,2]$ It is more likely to infiltrate the nasal cavity and maxillary sinus superiorly, which renders it difficult to determine the extent of the planned resection. It is rarely associated with cervical lymph node metastasis, as compared with mandibular gingival carcinoma. However, when it is associated with cervical lymph node metastasis, the prognosis is usually unfavourable. Moreover, patients with MGSCC show lower survival rates than those with mandibular gingival carcinoma.[3, 4] 
Therefore, the management of MGSCC is difficult, and the optimal treatment modality is still controversial.

An adequate number of reports have documented cases of combined MGSCC and palatal cancer.[5-7] However, limited number of sole MGSCC cases have been reported. Therefore, significant number of MGSCC cases have not been statistically analysed previously. Hence, we performed the statistical analysis of 90 MGSCC cases primarily treated in our department to clarify the clinical prognostic factors of MGSCC.

\section{Methods}

Ninety patients diagnosed with MGSCC and radically treated in our department between 1999 and 2014, consisting $36(40 \%)$ men and $54(60 \%)$ women with a mean age of 68.7 years (38-93 years) were included. Fifteen (16.7\%) patients were in the T1 stage, 32 (35.6\%) in T2, $13(14.4 \%)$ in T3, $22(24.4 \%)$ in $\mathrm{T} 4 \mathrm{a}$, and $8(8.9 \%)$ patients were in the T4b stage of MGSCC and $73(81.1 \%)$ patients were in the N0 stage, $4(4.4 \%)$ in N1, and $13(14.4 \%)$ were in the N2 stage of MGSCC, according to the 7th edition of the TNM classification. According to the classification of MGSCC by site of occurrence, proposed by Ojima et al., tumours extending to the soft palate, the medial or lateral pterygoid muscles, or the pterygoid process are categorised as the posterior region type, those confined around the anterior part as the anterior region type, and those in the molar region are categorised as the molar region type.[8] Of the 90 patients, 10 were anterior region cases (11\%), 53 were molar region (59\%), and 27 were posterior region cases (30\%). All study protocols were reviewed and approved by the institutional research ethics committee of our university. Informed consents were obtained from all participants in accordance with the institutional ethical guidelines. According to the approved principles of our department, we perform surgical treatment for early (T1 to early T2) and anterior region cancers. By contrast, for advanced (late stage T2 to T4) and posterior region cancers, multidisciplinary combination therapy with preoperative 5FU intra-arterial chemoradiotherapy (5FU $100 \sim 300 \mathrm{mg}$ for 21 days) with cannulation from the superficial temporal artery followed by surgical treatment in the form of maxillary resection and neck dissection is preferred.[9] The radiation dose is 40 to $50 \mathrm{~Gy}$ for primary lesions and 30 to $40 \mathrm{~Gy}$ for cervical lymph node metastasis. The maxillary resection line is determined preoperatively according to the tumour extension. Univariate analysis was performed to examine the significance of the following potential prognostic factors: age (< vs. $\geq 65$ years), site of occurrence (anterior or molar vs. posterior regions), degree of differentiation (well or moderate vs. poor), stage of tumour (T1 T4a vs. T4b), nodal metastasis (absence vs. presence), and treatment (only surgery or radiotherapy vs. combination of radiotherapy and surgery or combination of chemoradiotherapy and surgery). The SPSS 22.0 software (SPSS Inc., Chicago, IL) was used for the statistical analysis. Survival rates were calculated using the Kaplan-Meier method. Log rank tests and Chi-square tests were used to compare the survival rates between groups, and multivariate analysis was performed using the Cox proportional hazards model. The multivariate analysis was performed for prognostic factors with a cut-off value of $\mathrm{P}<0.25$ by the univariate analysis. The endpoint of univariate and multivariate analyses was the 5 -year overall survival rate. A P-value of less than 0.05 was defined as statistically significant. 


\section{Results}

According to the TNM classification, 15 patients (17\%) were in stage I, $29(32 \%)$ in stage II, $12(13 \%)$ in stage III, 25 (28\%) in stage IVA, and 9 cases (10\%) were in stage IVB. Forty-two patients were treated only by surgery, 5 only by radiotherapy, 3 by combination of radiotherapy and surgery, and 40 patients were treated by a multidisciplinary approach including combination of surgery following chemoradiotherapy. Histopathologically, 43 cases (47.8\%) were classified as well-differentiated, 39 (43.3\%) as moderately differentiated, and 8 cases (8.9\%) were classified as poorly differentiated. Cervical lymph node metastases were identified in 37 necks of 31 patients. Of these, neck dissection was not performed in 2 patients, because they had inoperable recurrences at other locations. Primary cervical lymph node metastases were evident in 13 cases, and secondary cervical lymph node metastases in 18 cases. Contralateral cervical lymph node metastases were found in 6 cases, and all were secondary metastases after initial neck dissection for the affected cervical lymph nodes. The number of lymph node metastases was 1 in 16 cases, 2 in 9 cases, 3 in 5 cases, 4 in 2 cases, and 5 in 3 cases, which was the maximum number of lymph node metastases found on one side of the neck. Extranodal extension was observed in 26 of 35 cases $(74.3 \%)$. The 5-year overall survival rate determined by the Kaplan-Meier method was $82.0 \%$. The disease-specific survival rate was $83.3 \%$. The overall survival rate for cases treated only by surgery $(91.5 \%, n=42)$ was better than that for those treated by combination of chemoradiotherapy and surgery $(79.0 \%, n=40)$; however, no statistically significant difference was observed. The 5-year overall survival rate according to tumour stage ( $\mathrm{T}$ staging) was $93.3 \%$ for $\mathrm{T} 1,88.9 \%$ for $\mathrm{T} 2,74.6 \%$ for $\mathrm{T} 3,76.4 \%$ for T4a, and $62.5 \%$ for T4b. Statistically significant differences were observed in survival rates between cases in T2 and T4b stages only. The 5-year overall survival rate according to the site of occurrence was $100 \%$ for the anterior region type, $87.4 \%$ for the molar region type, and $63.8 \%$ for the posterior region type (Fig. 1). The anterior region cancers showed significantly better survival rates than the posterior and molar region cancers. The molar region cancers showed significantly better survival rates than the posterior region cancers. The 5-year overall survival rate was significantly better $(88.1 \%)$ for the cervical lymph node-negative cases than that $(69.9 \%)$ for the cervical lymph node-positive cases including secondary metastasis. According to the degree of differentiation, the 5-year overall survival rate was $88.4 \%$ for well-differentiated, $76.3 \%$ for moderately differentiated, and $70.0 \%$ for poorly differentiated, with no statistically significant differences. The univariate analysis showed that the 5-year survival rates were significantly worse in patients with posterior region cancers and cervical lymph node-positive cases compared to the anterior and molar region cancers and cervical lymph node-negative cases, respectively (Table 1). The multivariate analysis demonstrated that site of cancer (posterior region) was the independent predictor of 5 -year overall survival rate $(P=0.031$, hazards ratio: $4.84,95 \%$ confidence interval: 1.01 to 10.18 ) (Table 2). 
Table 1

\begin{tabular}{|c|c|c|c|}
\hline \multicolumn{4}{|c|}{ Univariable analyses of prognostic factors for overall survival rate } \\
\hline & Number $(\mathrm{n}=90)$ & 5-y OS, \% & $\mathrm{p}$ value \\
\hline \multicolumn{4}{|l|}{ Age } \\
\hline$<65 y$ & 32 & 83.9 & 0.785 \\
\hline$\geqq 65 y$ & 58 & 80.9 & \\
\hline \multicolumn{4}{|l|}{ Site of occurrence } \\
\hline Anterior and Molar & 63 & 89.4 & $\star 0.003$ \\
\hline Posterior & 27 & 63.8 & \\
\hline \multicolumn{4}{|l|}{ Grade } \\
\hline well and moderate differentiated & 81 & 83.1 & 0.541 \\
\hline poor differentiated & 9 & 70.0 & \\
\hline \multicolumn{4}{|l|}{ Tumor(T)stage } \\
\hline $\mathrm{T} 1 \sim \mathrm{T} 4 \mathrm{a}$ & 82 & 84.0 & 0.074 \\
\hline $\mathrm{T} 4 \mathrm{~b}$ & 8 & 62.5 & \\
\hline \multicolumn{4}{|l|}{$\mathrm{pN}$} \\
\hline negative & 59 & 88.1 & $\star 0.015$ \\
\hline positive & 31 & 69.9 & \\
\hline \multicolumn{4}{|l|}{ Treatment } \\
\hline $\mathrm{S}, \mathrm{Ra}$ & 47 & 87.9 & 0.120 \\
\hline$R a+S, C+R a+S$ & 43 & 75.7 & \\
\hline
\end{tabular}


Table 2

\begin{tabular}{|c|c|c|c|}
\hline \multicolumn{4}{|c|}{ Multivariable analyses of prognostic factors for overall survival rate } \\
\hline & HR & $95 \% \mathrm{Cl}$ & $\mathrm{p}$ value \\
\hline \multicolumn{4}{|l|}{ Site of occurrence } \\
\hline Anterior and Molar & 3.30 & $1.11-9.79$ & *0.031 \\
\hline \multicolumn{4}{|l|}{ Posterior } \\
\hline \multicolumn{4}{|l|}{ Tumor(T)stage } \\
\hline $\mathrm{T} 1 \sim \mathrm{T} 4 \mathrm{a}$ & 1.44 & $0.36-5.79$ & 0.607 \\
\hline \multicolumn{4}{|l|}{$\mathrm{T} 4 \mathrm{~b}$} \\
\hline \multicolumn{4}{|l|}{ pN } \\
\hline Negative & 2.58 & $0.91-7.35$ & 0.076 \\
\hline \multicolumn{4}{|l|}{ positive } \\
\hline \multicolumn{4}{|l|}{ Treatment } \\
\hline S, Ra & 1.80 & $0.59-5.49$ & 0.301 \\
\hline \multicolumn{4}{|l|}{$\mathrm{Ra}+\mathrm{S}, \mathrm{CRT}+\mathrm{S}$} \\
\hline $\mathrm{HR}=\mathrm{Hazard}$ ratio, $\mathrm{C}$ & & & \\
\hline
\end{tabular}

\section{Discussion}

MGSCC is relatively rare, and few reports have analysed the treatment strategies and prognostic factors in a significant number of cases.[10] In addition, since palatal cancer involving the hard palate and MGSCC show similar clinical findings, some reports have analysed the combination, but few reports have analysed only MGSCC.[11-16] This study analysed the management methods and prognostic factors of MGSCC in 90 cases. Eskander at al. reported that poor differentiation in tumours was an independent predictor of disease-specific survival rate.[14] Poeschl et al. reported that T staging and grading do not have a significant impact on long-term survival of patients.[6] Yang et al. reported statistically significant associations among patient survival rate and tumour differentiation grade, $T$ staging, cervical lymph node metastasis, and local recurrence.[15] Ojima et al. reported that the posterior region cancers showed poor prognoses compared with the anterior and molar region cancers.[8] In the multivariate analysis, site of occurrence contributed significantly to the survival rate in our study. Posterior region MGSCCs showed significantly poor prognoses compared with the anterior and molar region types. Nine of 20 cases with poor prognoses showed locoregional recurrence after the primary surgery, while 5 of the 9 cases were posterior region cancers. It is suggested that posterior region cancers show poor prognoses due to the 
difficulties encountered in including sufficient safe areas in resection, in spite of the surgical margins determined as negative.

Prophylactic neck dissection is recommended in cases of cervical lymph node metastasis with MGSCC. [17-21] In our study, 8 patients in the N0 stage underwent prophylactic neck dissection, and 64 patients were closely monitored without any treatment. One patient treated with prophylactic neck dissection developed cervical lymph node metastasis. In recent years, several reports have recommended prophylactic neck dissection even in early stages.[1, 20,22] Moratin et al. suggested the concept of prophylactic neck dissection in early tumours with clinically negative neck status.[22] Poeschl et al. reported that prophylactic neck dissection did not significantly improve overall survival rates and did not prevent the rate of regional recurrence in cNO-staged patients with MGSCC and palatal SCC.[6] In our study, 11 of the 47 cases in T1 and T2 stages (23\%) developed postoperative cervical lymph node metastases, which were treated by neck dissection. The 5-year overall survival rate in these patients was $80 \%$, indicating that neck dissection even after subsequent cervical metastasis is very effective.

Therefore, we suggest that NO-staged MGSCC patients should be closely monitored, and neck dissection should be performed immediately upon the onset of cervical lymph node metastasis.

The overall survival rates of patients with MGSCC have been previously reported as $24-71 \% .[6,15,20$, 23] Binahmed et al. reported the 5-year overall survival rate as 33\%.[13] Yang et al reported the 5-year overall survival rate as $57.5 \%$.[15] A critical comparison could not performed because their analysis included carcinomas of the hard palate. Morice et al. reported the 5 -year overall survival rate as $32 \%$ for MGSCC. In their analysis of 47 patients, 12 cases were in the T1 to T3 stages (26\%), and 35 were in the T4 stage (74\%). In contrast, our study revealed the 5 -year overall survival rate as $82.1 \%$. In our study, 60 cases were in the T1 to T3 stage (66.7\%), while 30 cases were in the T4 stage (33.3\%). Hence, the lower survival rate in the study by Morice et al. could be attributed to the greater number of advanced cases. However, even the 5-year disease-specific survival rate (76.7\%) of cases in advanced T4a stage in this study was better than that of corresponding cases $(54 \%)$ in their study. The better survival rate in our study could be a result not only of the smaller number of advanced cases but also of better results for the advanced cases. Morice et al. and Yang et al. treated most patients surgically. However, we performed combination therapy including surgery and chemoradiotherapy for advanced cases. This could be the reason for better survival rates even in the advanced cases of our study.

Twenty patients died during the study period, including 3 deaths due to other diseases and 17 due to MGSCC (T1: 2 cases, T2: 6 cases, T3: 3 cases, T4a: 6 cases, and T4b: 3 cases). Seven patients died in primary recurrence, 2 patients in cervical recurrence, one in primary recurrence with lateral retropharyngeal (Rouviere) lymph node metastasis, one in primary and cervical recurrences, and 6 patients died in distant metastases including 2 patients with untreatable primary and cervical recurrence with Rouviere lymph node metastasis. Of the 17 deaths due to the MGSCC, 6 deaths (35.3\%) occurred due to distant metastases, suggesting that management of distant metastasis is particularly important for improving future treatment outcomes. 


\section{Conclusions}

We reported 90 cases of MGSCC. We observed that posterior region cancers, according to the classification based on site of occurrence, were independent predictors of poor 5-year overall survival rate. Further long-term studies with larger sample sizes should be performed to analyse the results of various treatment modalities for MGSCC.

\section{Abbreviations}

MGSCC

Maxillary gingibal squamous cell carcinoma

TNM

tumour, node, metastasis

\section{Declarations}

\section{Ethics approval and consent to participate}

This retrospective study was approved by the Research Ethics Committee of Tokyo Medical and Dental University (Registry No. D2013-034) and written informed consent was waived because of its retrospective design.

\section{Consent for publication}

Not applicable

\section{Competing interests}

None

\section{Funding}

None

\section{Authors' contributions}

All authors contributed to the study conception and design. YO and MY analyzed the data and wrote the manuscript. CM, MM KM, IS and DY collected the data. YM, NU, JS, KK and TY collected the data and wrote the manuscript. All authors have read and approved the final manuscript.

\section{Acknowledgments}

None 
The datasets generated during the current study are not publicly available since they will contain patient data and the informed consent agreement does not include sharing data publicly.

\section{References}

1. Berger $M$, Grau $E$, Saure $D$, et al. Occurrence of cervical lymph node metastasis of maxillary squamous cell carcinoma - A monocentric study of 171 patients. J Craniomaxillofac Surg. Dec 2015;43(10):2195-9. doi:10.1016/j.jcms.2015.09.009

2. Philip J, James R. Maxillary squamous cell carcinoma: an 11-year retrospective study of one regional cancer centre. Int J Oral Maxillofac Surg. Oct 2014;43(10):1195-8. doi:10.1016/j.ijom.2014.05.020

3. Muñoz Guerra MF, Naval Gías L, Campo FR, Pérez JS. Marginal and segmental mandibulectomy in patients with oral cancer: a statistical analysis of 106 cases. J Oral Maxillofac Surg. Nov 2003;61(11):1289-96. doi:10.1016/s0278-2391(03)00730-4

4. Nassiri AM, Campbell BR, Mannion K, Sinard RJ, Netterville JL, Rohde SL. Survival Outcomes in T4aNOMO Mandibular Gingival Squamous Cell Carcinoma Treated with Surgery Alone. Otolaryngol Head Neck Surg. 05 2019;160(5):870-875. doi:10.1177/0194599818821892

5. Beltramini GA, Massarelli O, Demarchi $M$, et al. Is neck dissection needed in squamous-cell carcinoma of the maxillary gingiva, alveolus, and hard palate? A multicentre Italian study of 65 cases and literature review. Oral Oncol. Feb 2012;48(2):97-101.

doi:10.1016/j.oraloncology.2011.08.012

6. Poeschl PW, Russmueller G, Seemann R, et al. Staging and grading as prognostic factors in maxillary squamous cell carcinoma. J Oral Maxillofac Surg. Dec 2011;69(12):3038-44.

doi:10.1016/j.joms.2011.02.064

7. Mourouzis $C$, Pratt $C$, Brennan PA. Squamous cell carcinoma of the maxillary gingiva, alveolus, and hard palate: is there a need for elective neck dissection? Br J Oral Maxillofac Surg. Jul 2010;48(5):345-8. doi:10.1016/j.bjoms.2009.07.012

8. Yasutaka O, Masahiro U, Hideki K, et al. A clinicopathological study of 49 patients with squamous cell carcinoma of the upper gingiva. Japanese Journal of Oral \& Maxillofacial Surgery. 2006;52(9):6.

9. Masashi Y, Yoshio O, Yasuyuki M, et al. A clinicopathological analysis of upper gingival cancer. Japanese Journal of Head and Neck Cancer. 2011;37(1):6.

10. Slieker FJB, de Bree R, Van Cann EM. Predicting individualized mortality probabilities for patients with squamous cell carcinoma of the maxilla: Novel models with clinical and histopathological predictors. Head Neck. Jul 2019;doi:10.1002/hed.25879

11. Wang TC, Hua CH, Lin CC, Tsou YA, Tseng HC, Tsai MH. Risk factors affect the survival outcome of hard palatal and maxillary alveolus squamous cell carcinoma: 10-year review in a tertiary referral center. Oral Surg Oral Med Oral Pathol Oral Radiol Endod. Jul 2010;110(1):11-7. doi:10.1016/j.tripleo.2009.11.035 
12. Morris LG, Patel SG, Shah JP, Ganly I. High rates of regional failure in squamous cell carcinoma of the hard palate and maxillary alveolus. Head Neck. Jun 2011;33(6):824-30. doi:10.1002/hed.21547

13. Binahmed A, Nason RW, Hussain A, Abdoh AA, Sándor GK. Treatment outcomes in squamous cell carcinoma of the maxillary alveolus and palate: a population-based study. Oral Surg Oral Med Oral Pathol Oral Radiol Endod. Jun 2008;105(6):750-4. doi:10.1016/j.tripleo.2007.09.001

14. Eskander A, Givi B, Gullane PJ, et al. Outcome predictors in squamous cell carcinoma of the maxillary alveolus and hard palate. Laryngoscope. Oct 2013;123(10):2453-8. doi:10.1002/lary.24079

15. Yang X, Song X, Chu W, Li L, Ma L, Wu Y. Clinicopathological Characteristics and Outcome Predictors in Squamous Cell Carcinoma of the Maxillary Gingiva and Hard Palate. J Oral Maxillofac Surg. Jul 2015;73(7):1429-36. doi:10.1016/j.joms.2014.12.034

16. Montes DM, Schmidt BL. Oral maxillary squamous cell carcinoma: management of the clinically negative neck. J Oral Maxillofac Surg. Apr 2008;66(4):762-6. doi:10.1016/j.joms.2007.12.017

17. Dalal AJ, McLennan AS. Cervical metastases from maxillary squamous cell carcinoma: retrospective analysis and review of the literature. Br J Oral Maxillofac Surg. Dec 2013;51(8):702-6. doi:10.1016/j.bjoms.2013.08.011

18. Zhang WB, Peng X. Cervical metastases of oral maxillary squamous cell carcinoma: A systematic review and meta-analysis. Head Neck. 04 2016;38 Suppl 1:E2335-42. doi:10.1002/hed.24274

19. Zhang WB, Wang Y, Mao C, Guo CB, Yu GY, Peng X. Cervical metastasis of maxillary squamous cell carcinoma. Int J Oral Maxillofac Surg. Mar 2015;44(3):285-91. doi:10.1016/j.ijom.2014.10.020

20. Tang $L$, Leung $Y Y$. When should elective neck dissection be performed in maxillary gingival and alveolar squamous cell carcinoma with a cNO neck? A systematic review. Int J Oral Maxillofac Surg. Nov 2016;45(11):1358-1365. doi:10.1016/j.ijom.2016.05.021

21. Poeschl PW, Seemann R, Czembirek C, et al. Impact of elective neck dissection on regional recurrence and survival in cNO staged oral maxillary squamous cell carcinoma. Oral Oncol. Feb 2012;48(2):1738. doi:10.1016/j.oraloncology.2011.09.006

22. Moratin J, Fuchs A, Zeidler C, et al. Squamous cell carcinoma of the maxilla: Analysis of clinicopathological predictors for disease recurrence and metastatic behavior. J Craniomaxillofac Surg. Apr 2018;46(4):611-616. doi:10.1016/j.jcms.2018.01.002

23. Morice A, Ostertag A, Sahli-Amor M, Goudot P, Bertolus C, Schouman T. Prognostic factors of gingival-alveolar squamous cell carcinoma of the maxilla. Surg Oncol. Sep 2016;25(3):263-8. doi:10.1016/j.suronc.2016.05.011

\section{Figures}




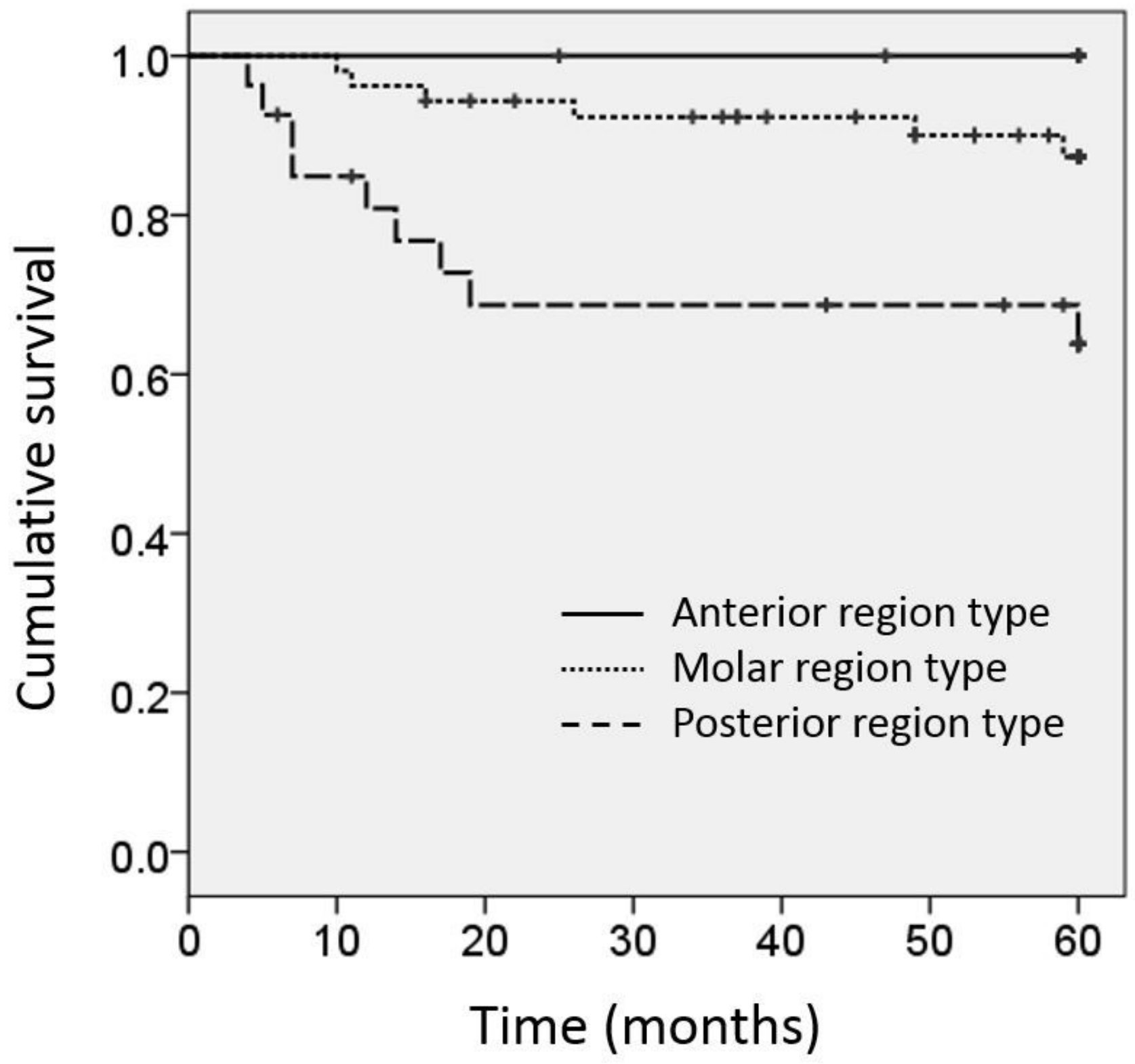

Figure 1

Kaplan-Meier curve. Five-year overall survival rate of patients with molar region maxillary gingival squamous cell carcinoma was significantly better than that of patients with posterior region carcinoma $(P<0.05)$. 


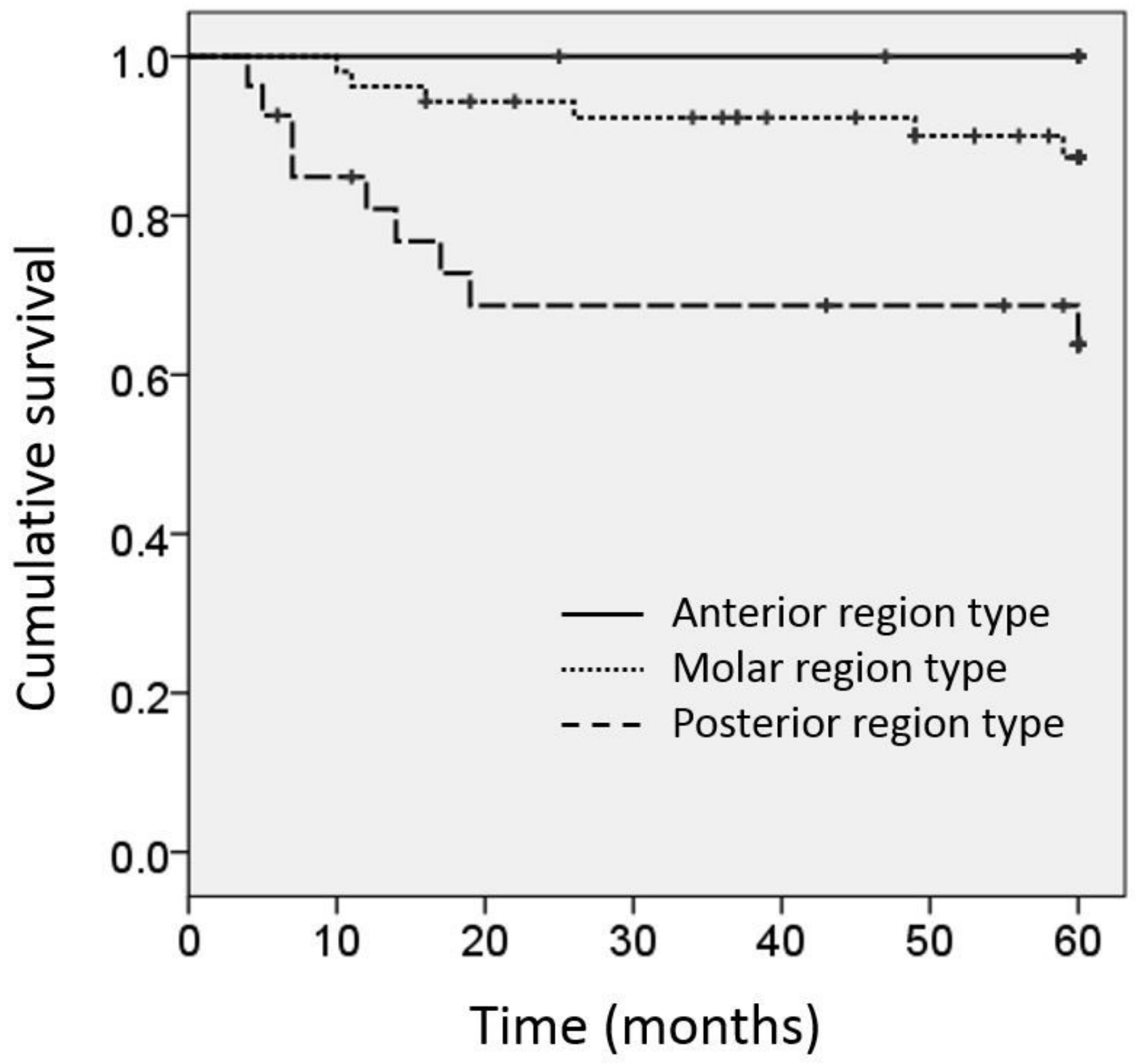

Figure 1

Kaplan-Meier curve. Five-year overall survival rate of patients with molar region maxillary gingival squamous cell carcinoma was significantly better than that of patients with posterior region carcinoma $(P<0.05)$. 